\title{
ESTUDO ATRAVÉS DA RESSONÂNCIA MAGNÉTICA DE 67 CASOS DE GLIOBLASTOMA MULTIFORME E A OCORRÊNCIA DE METÁSTASES
}

\author{
Nelson Fortes Ferreira1', Marcelo Barbosa², Lazaro L. Faria do Amaral', \\ Renato Adam Mendonça', Sérgio Santos Lima
}

\begin{abstract}
RESUMO - Objetivo: Demonstrar as características de imagem do glioblastoma multiforme, tumor primário mais comum do sistema nervoso central, com ênfase em suas localizações, ocorrência de metástases e relação com o grau de necrose demonstrado por ressonância magnética (RM). Método: Foram analizados retrospectivamente 67 casos de ressonância magnética de pacientes com diagnóstico histológico de glioblastoma multiforme, retirados do arquivo didático da MED IMAGEM. Todos os exames foram realizados em aparelhos GE de 1,5 T. (GE- Medical Systems), no período compreendido entre janeiro de 1995 e maio de 2003. Resultados: Nos 67 casos estudados, a idade dos pacientes variou entre 4 e 86 anos, com idade média de 60 anos. Houve predominância no sexo masculino, com 39 casos (58\%). A localização frontal foi preponderante (47\%) seguida de $18 \%$ na região temporal e $16 \%$ na região parietal. Em $19 \%$ se localizou em outros sítios. $O$ aparecimento de metástases ocorreu em 15 casos. Conclusão: Concordante com a literatura o glioblastoma multiforme predomina nos lobos frontais e acomete preferencialmente pacientes com idade acima de 50 anos. 0 aparecimento de metástases em nossos casos ocorreu em $22 \%$.
\end{abstract}

PALAVRAS-CHAVE: glioblastoma multiforme, necrose, ressonância magnética, metástases.

\begin{abstract}
Magnetic resonance imaging in 67 cases of glioblastoma multiforme and occurrence of metastases
ABSTRACT - Purpose: The purpose of this paper is to demonstrate the main MRI characteristics of glioblastoma multiforme (GBM), the most common CNS primary tumor, emphasizing its location and the occurrence of metastases. Method: The MR imaging of 67 pathologically proven cases of glioblastoma multiforme were retrospectively reviewed. The exams were realized in the period between 1995 and 2003, in one of three 1.5 Signa GE units (Milwaukee, WI). Results: The ages of the patients ranged from 4 years to 86 years, mean 60 years, and the occurrence of the tumor was preponderant among men, with 39 cases (58\%). The most common location was in the frontal lobes (47\%) followed by the temporal lobes (18\%) and the parietal lobes (16\%). In $19 \%$ of the cases there were involvement of more than one site and long distance metastases were seen in $22 \%$ of the patients. Conclusion: According to the literature, the most common location of GBM was in the frontal lobe of older than 50 years old men. Metastases occurred in $22 \%$ of our cases.
\end{abstract}

KEY WORDS: glioblastoma multiforme, necrosis, magnetic resonance imaging, metastases.

As características do glioblastoma multiforme (GBM) estudado por imagens de ressonância magnética (RM) são bem conhecidas e demonstradas na literatura atual. Apesar de ter prevalência definida, curso rápido e fatal, esta neoplasia pode comprometer diferentes localizações e determinar a ocorrência de implantes secundários ${ }^{1-3}$. As imagens obtidas por RM do sistema nervoso central (SNC) em seus diversos planos e seqüências tem se mostrado um ótimo método para avaliação deste tumor $^{1-3}$. Análisamos neste artigo, 67 casos de GBM, ressaltando a incidência de metástases e tentando correlacionar este fato com o grau de necrose do tumor.

\section{MÉTODO}

Foram avaliados retrospectivamente 67 exames de RM realizados na MED IMAGEM (Tabela 1) Hospital Beneficência Portuguesa de São Paulo, em pacientes com diagnóstico histológico de GBM. Classificamos estes tumores em relação ao grau de necrose de acordo com a classificação de Hammoud ${ }^{4}$ (Fig 1). Os exames de RM foram realizados em aparelhos $\mathrm{GE}$ de alto campo $(1,5 \mathrm{~T})$.

MED IMAGEM - Hospital Beneficência Portuguesa de São Paulo, São Paulo SP, Brasil: ${ }^{1}$ Neuroradiologista; ${ }^{2}$ Médico Estagiário; ${ }^{3} \mathrm{Chefe}$ do Serviço de Radiologia

Recebido 1 Dezembro 2003, recebido na forma final 4 Março 2004. Aceito 8 Abril 2004.

Dr. Nelson Fortes Ferreira - Rua Martiniano de Carvalho 611/123 - 01321-001 São Paulo SP - Brasil. E-mail: neldiz@hotmail.com 
Tabela 1 Correlação dos pacientes com idade, sexo, local de acometimento, necrose, edema e local de metástase.

\begin{tabular}{|c|c|c|c|c|c|c|}
\hline Paciente & Idade & Sexo & Local de acometimento & Necrose & Edema & Local de metástase \\
\hline 1 & 49 & M & Lobo Parietal & Grau III & Moderado & \\
\hline 2 & 72 & M & Lobo Occipital & Grau III & Acentuado & Invasão sub-ependimária \\
\hline 3 & 55 & M & Lobo Occipital Bilateral & Grau III & Moderado & Invasão sub - ependimária \\
\hline 4 & 21 & M & Lobo Frontal & Grau III & Acentuado & Sub - ependimária \\
\hline 5 & 77 & $\mathrm{~F}$ & Lobo Parietal & Grau III & Moderado & \\
\hline 6 & 22 & M & Lobo Temporal & Grau III & Discreto & $\begin{array}{l}\text { Drop - metástase e metástase } \\
\text { para corpo vertebral }\end{array}$ \\
\hline 7 & 35 & $\mathrm{~F}$ & Temporo - Parietal & Grau II & Discreto & Drop - metástases \\
\hline 8 & 41 & M & Lobo Temporal & Grau II & Discreto & Metástase para pescoço \\
\hline 9 & 29 & M & Tronco Cerebral & Grau 0 & Sem & Drop - metástase \\
\hline 10 & 42 & M & Corpo Caloso & Grau I & Moderado & Invasão sub - ependimária \\
\hline 11 & 31 & M & Lobo Frontal & Grau II & Sem & Metástase contra lateral \\
\hline 12 & 66 & $\mathrm{~F}$ & Lobo Temporal & Grau I & Acentuado & Drop - metástase \\
\hline 13 & 34 & $\mathrm{~F}$ & Frontal Bilateral & Grau III & Acentuado & Drop - metástase \\
\hline 14 & 58 & M & Frontal + Temporal & Grau I & Acentuado & \\
\hline 15 & 35 & M & Lobo Temporal & Grau II & Acentuado & Drop - metástase \\
\hline 16 & 54 & $\mathrm{~F}$ & Lobo Frontal & Grau I & Acentuado & \\
\hline 17 & 36 & $\mathrm{~F}$ & Lobo Frontal & Grau I & Discreto & \\
\hline 18 & 25 & M & Lobo Temporal & Grau II & Discreto & \\
\hline 19 & 31 & $\mathrm{~F}$ & Lobo Frontal & Grau III & Acentuado & \\
\hline 20 & 86 & $\mathrm{~F}$ & Parieto occipital & Grau 0 & Acentuado & \\
\hline 21 & 53 & M & Lobo Frontal & Grau II & Moderado & \\
\hline 22 & 51 & M & Lobo Frontal Direito & Grau III & Moderado & \\
\hline 23 & 47 & M & Lobo Occipital & Grau 0 & Discreto & \\
\hline 24 & 57 & M & Lobo Frontal & Grau 0 & Moderado & \\
\hline 25 & 62 & M & Lobo Temporal & Grau I & Acentuado & \\
\hline 26 & 68 & $\mathrm{~F}$ & Lobo Parietal & Grau 0 & Acentuado & \\
\hline 27 & 49 & M & Parieto occipital + corpo caloso & Grau I & Acentuado & \\
\hline 28 & 73 & M & Lobo Temporal & Grau I & Acentuado & \\
\hline 29 & 61 & $\mathrm{~F}$ & Lobo Frontal & Grau 0 & Acentuado & \\
\hline 30 & 28 & $\mathrm{~F}$ & Lobo Frontal & Grau 0 & Acentuado & \\
\hline 31 & 46 & $\mathrm{~F}$ & Lobo Parietal & Grau I & Acentuado & \\
\hline 32 & 35 & $\mathrm{~F}$ & Lobo Frontal & Grau I & Acentuado & \\
\hline 33 & 81 & $\mathrm{~F}$ & Corpo Caloso & Grau I & Moderado & \\
\hline 34 & 52 & $\mathrm{~F}$ & Lobo Frontal Bilateral & Grau II & Acentuado & \\
\hline 35 & 46 & M & Lobo Temporal & Grau 0 & Discreto & \\
\hline 36 & 67 & $\mathrm{~F}$ & Lobo Parietal & Grau I & Acentuado & Invasão sub - ependimária \\
\hline 37 & 36 & M & Lobo Frontal & Grau II & Moderado & Invasão sub - ependimária \\
\hline 38 & 45 & M & Lobo Frontal & Grau II & Moderado & \\
\hline 39 & 40 & M & Lobo Frontal & Grau I & Discreto & \\
\hline 40 & 55 & M & Lobo Temporal & Grau I & Moderado & Invasão sub - ependimária \\
\hline 41 & 50 & $\mathrm{~F}$ & Lobo Frontal & Grau III & Acentuado & \\
\hline 42 & 68 & M & Corpo Caloso & Grau I & Discreto & \\
\hline 43 & 57 & M & Lobo Frontal & Grau II & Discreto & \\
\hline 44 & 45 & $\mathrm{~F}$ & Lobo Parietal & Grau III & Discreto & \\
\hline 45 & 65 & M & Lobo Frontal & Grau II & Moderado & \\
\hline 46 & 68 & $\mathrm{~F}$ & Lobo Frontal & Grau II & Acentuado & \\
\hline 47 & 62 & M & Lobo Parietal & Grau I & Discreto & \\
\hline 48 & 85 & M & Lobo Temporal & Grau II & Acentuado & \\
\hline 49 & 62 & M & Lobo Parietal & Grau 0 & Discreto & \\
\hline 50 & 60 & $\mathrm{~F}$ & Lobo Parietal & Grau II & Moderado & \\
\hline 51 & 28 & $\mathrm{~F}$ & Lobo Frontal & Grau III & Acentuado & \\
\hline 52 & 66 & $M$ & Lobo Frontal & Grau I & Acentuado & \\
\hline 53 & 34 & M & Lobo Frontal & Grau III & Acentuado & \\
\hline 54 & 30 & $\mathrm{~F}$ & Lobo Parietal & Grau II & Acentuado & \\
\hline 55 & 44 & $\mathrm{~F}$ & Lobo Temporal & Grau III & Acentuado & \\
\hline 56 & 33 & $\mathrm{~F}$ & Lobo Frontal & Grau 0 & Sem & \\
\hline 57 & 71 & $\mathrm{~F}$ & Lobo Frontal & Grau II & Moderado & \\
\hline 58 & 24 & $M$ & Lobo Frontal & Grau I & Discreto & \\
\hline 59 & 69 & $\mathrm{~F}$ & Lobo Occipital & Grau III & Moderado & \\
\hline 60 & 41 & $\mathrm{~F}$ & Lobo Frontal & Grau III & Moderado & \\
\hline 61 & 56 & M & Lobo Temporal & Grau I & Moderado & \\
\hline 62 & 51 & M & Lobo Frontal & Grau I & Moderado & \\
\hline 63 & 70 & $\mathrm{~F}$ & Lobo Frontal & Grau I & Moderado & \\
\hline 64 & 30 & $M$ & Lobo Frontal & Grau I & Acentuado & \\
\hline 65 & 56 & M & Lobo Frontal & Grau I & Moderado & \\
\hline 66 & 04 & $\mathrm{~F}$ & Lobo Parietal & Grau I & Moderado & \\
\hline 67 & 48 & $\mathrm{M}$ & Lobo Frontal & Grau I & Moderado & \\
\hline
\end{tabular}




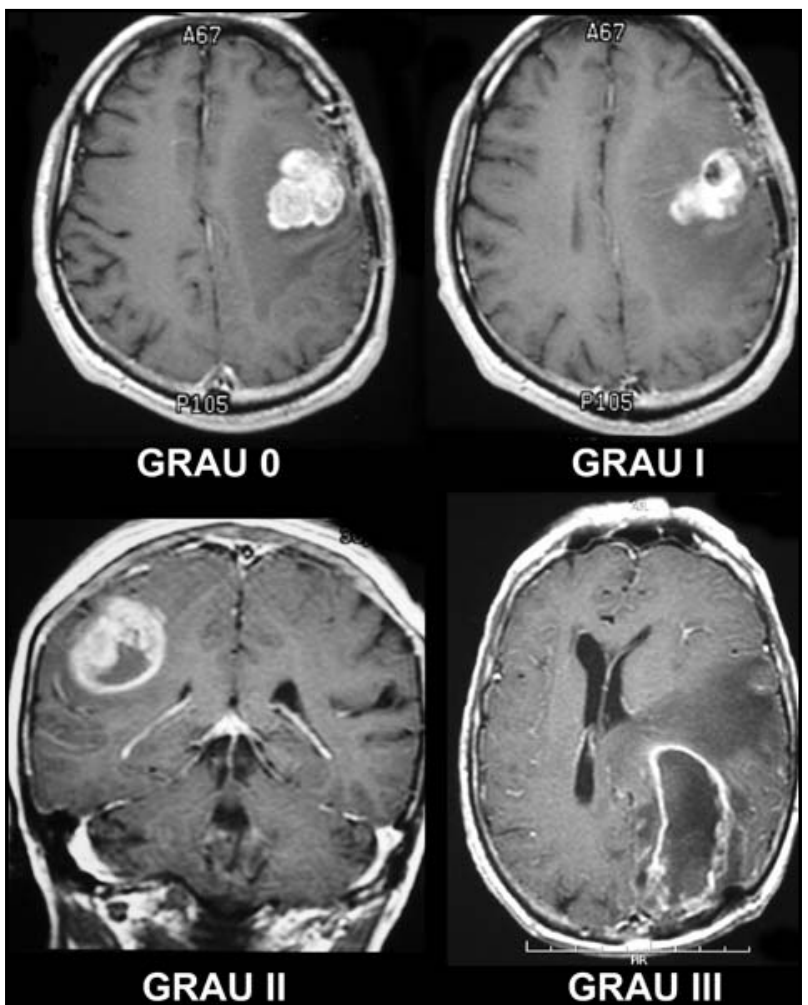

Fig 1. Graus de necrose segundo Hammoud.

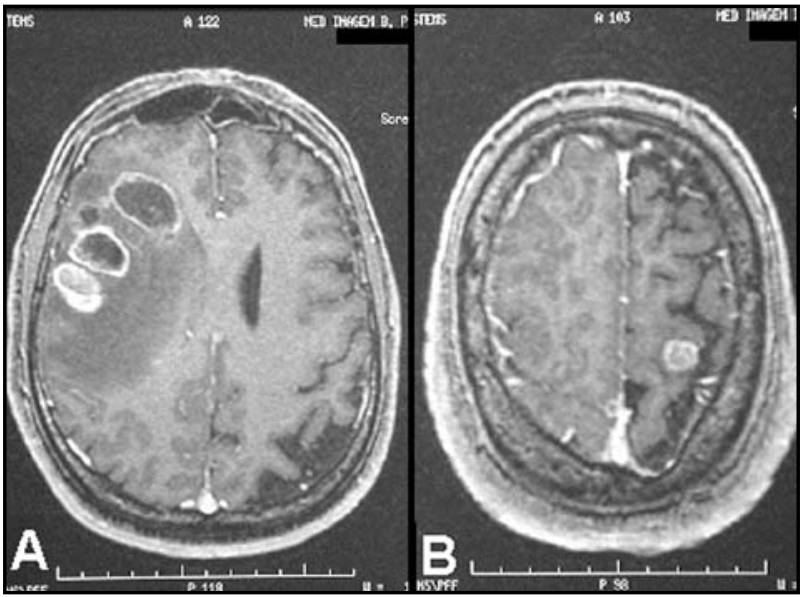

Fig 2. A. Axial T1 pós Gd-DTPA - GBM frontal direito. B. Metástase para alta convexidade parietal esquerda.

Foram consideradas lesões metastásicas aquelas que apareceram na região sub-ependimária, nas meninges distantes do tumor inicial, no canal raqueano, fora do sistema nervoso central (SNC) ou lesões parenquimatosas distantes da lesão inicial, sem ligação visível pela RM e com aparecimento de pelo menos 3 meses após a visibilização da lesão primária.

\section{RESULTADOS}

A idade média dos pacientes estudados foi 48 anos, variando entre 4 e 86 anos; em metade dos

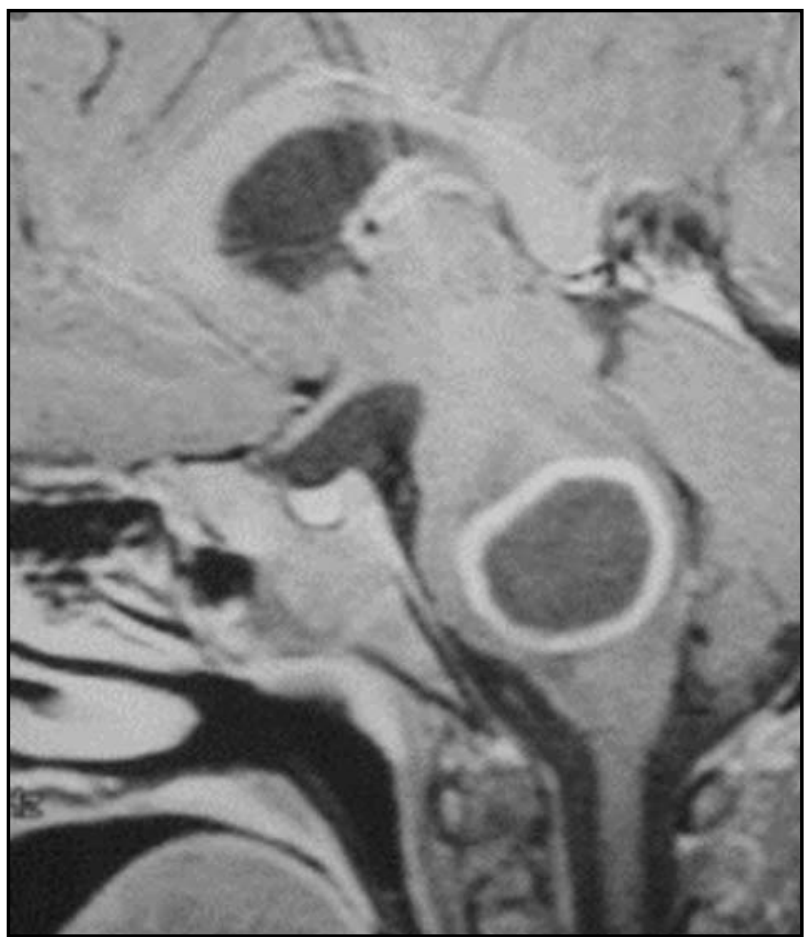

Fig 3. Sagital T1 pós Gd-DTPA - GBM de tronco.

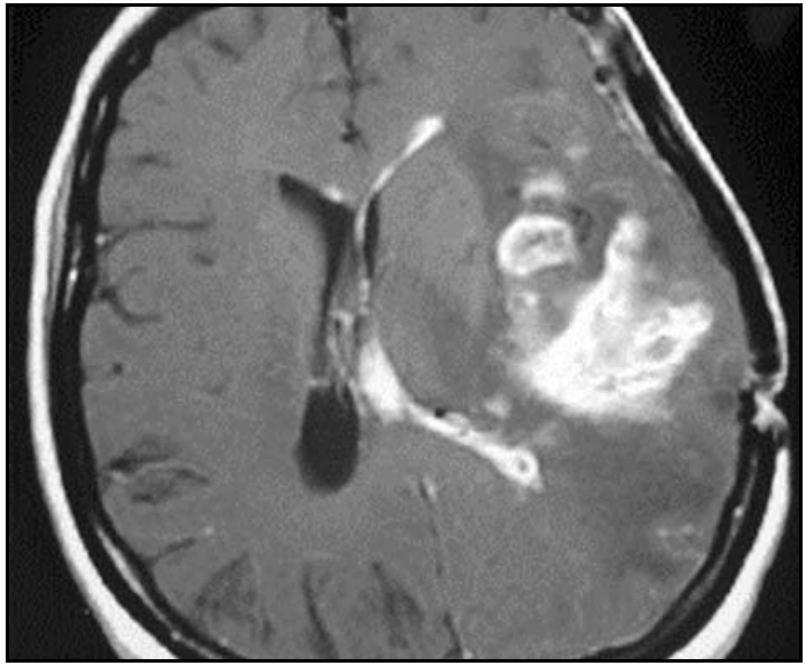

Fig 4. Axial T1 pós Gd-DTPA GBM fronto-parietal esquerdo com efeito de massa e disseminação para ventrículo lateral esquerdo.

pacientes o diagnóstico foi feito entre 50 e 70 anos de idade (Tabela 1). O lobo frontal foi o mais acometido (Fig 2) com $47 \%$ dos casos, seguido do lobo temporal (18\%) e lobo parietal (16\%).

Em 19\% dos casos evidenciamos outros locais envolvidos ou 2 localizações associadas.

Entre as localizações menos habituais encontramos: lobo occipital (6 casos); corpo caloso (8 casos); tronco cerebral (1 caso) (Fig 3).

Metástases ocorreram em 15 pacientes (16 regiões) $(22 \%)$, sendo os locais de disseminação as 


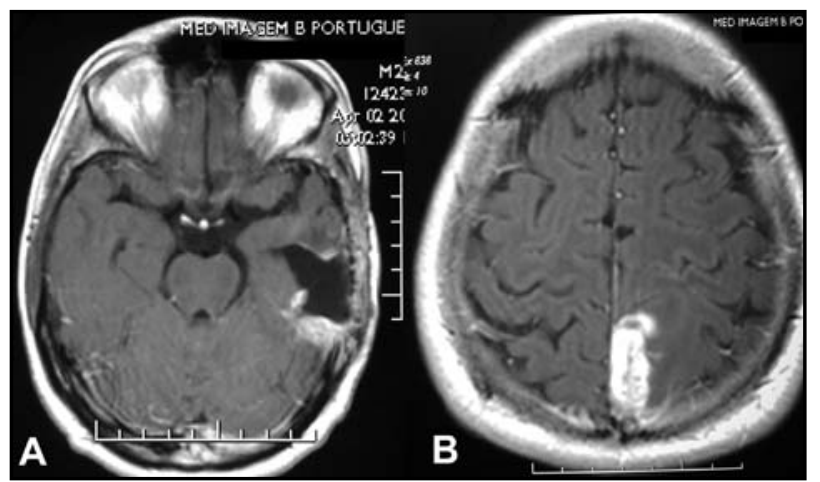

Fig 5 A. Cavidade cirúrgica da lesão primária. B. Implante secundário da lesão inicial (confirmado cirurgicamente).

que seguem (Gráfico 1): disseminação subependimária/ Invasão leptomeninges (Fig 4); metástase parenquimatosa (Fig 5); disseminação extracraniana para pescoço (Fig 6); "drop" metástases (Fig 7); metástase para corpo vertebral (Fig 8).

Nos tumores em que ocorreram metástases os graus de necrose observados foram preferencialmente II (24\%) e III (24\%) (Gráfico 2).

\section{DISCUSSÃO}

O GBM é a neoplasia de mais alto grau na classificação dos tumores astrocíticos. É o tumor primário do SNC mais comum no adulto ${ }^{1,2,5}$.

Alguns GBM podem originar-se de astrocitomas de baixo grau, embora outros não demonstrem esta relação (glioblastoma multiforme "de novo") ${ }^{1,2,5}$.

Geralmente, acomete homens em torno de 50 anos de idade, sendo menos freqüentes abaixo dos 30 anos. Apresenta-se tipicamente com sintomas clínicos relacionados a hipertensão intracraniana ou convulsões ${ }^{1,5}$.

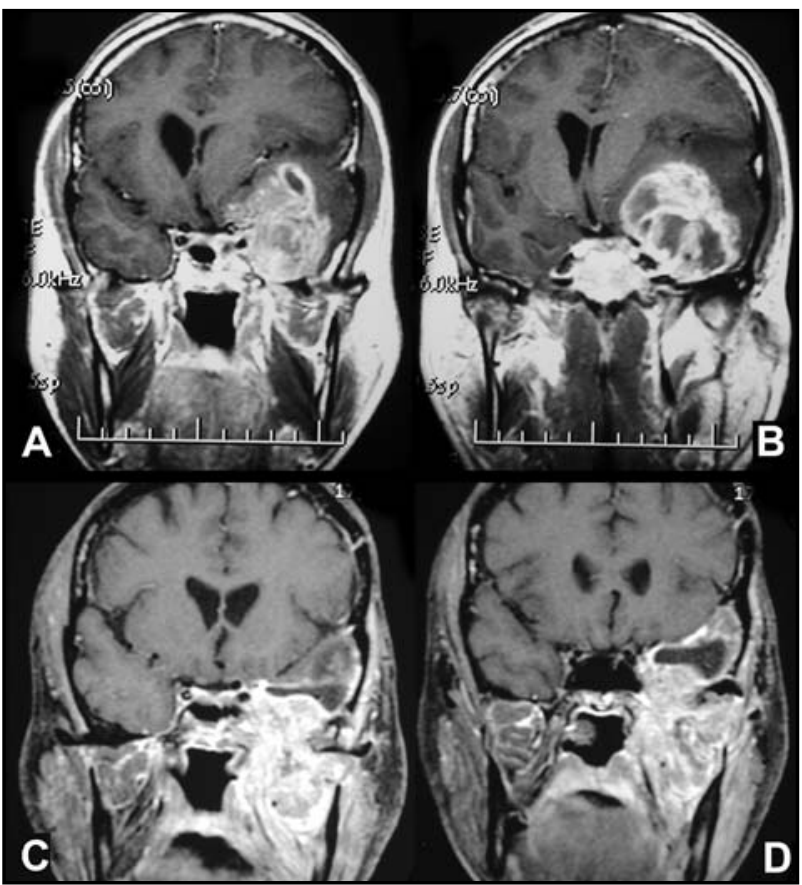

Fig 6. Coronal T1 pós Gd-DTPA mostrando volumoso GBM temporal esquerdo com efeito de massa deslocando as estruturas da linha média. Controle evolutivo mostrando exérese parcial da lesão intracraniana e disseminação para o pescoço.

Quanto à localização, são mais comuns nos lobos frontal e temporal, podendo envolver mais de um lobo ${ }^{1,2,4}$. Em crianças, a localização preferencial é infratentorial, correspondendo à quarta neoplasia mais comum abaixo dos dois anos de idade ${ }^{1,2,6}$. As características de imagem do GBM na RM são bem descritas na literatura como massa mal delimitada, com sinal heterogêneo em todas as seqüências, áreas císticas/necrose, com paredes irregulares e impregnação heterogênea pelo contraste ${ }^{1,2,5}$. As áreas de necrose são características de neoplasias de alto

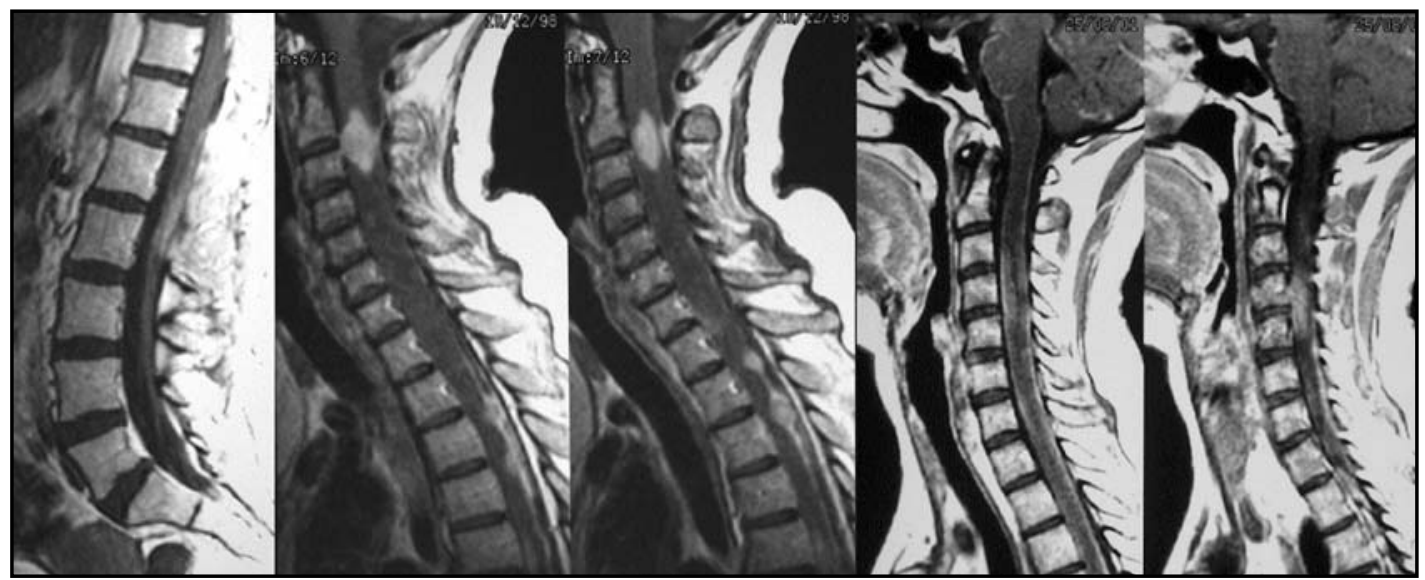

Fig 7. Sequências sagitais $T 1$ pós Gd-DTPA mostrando múltiplas lesões nodulares em situação intradural e extramedular compatíveis com "drop" metástases. 


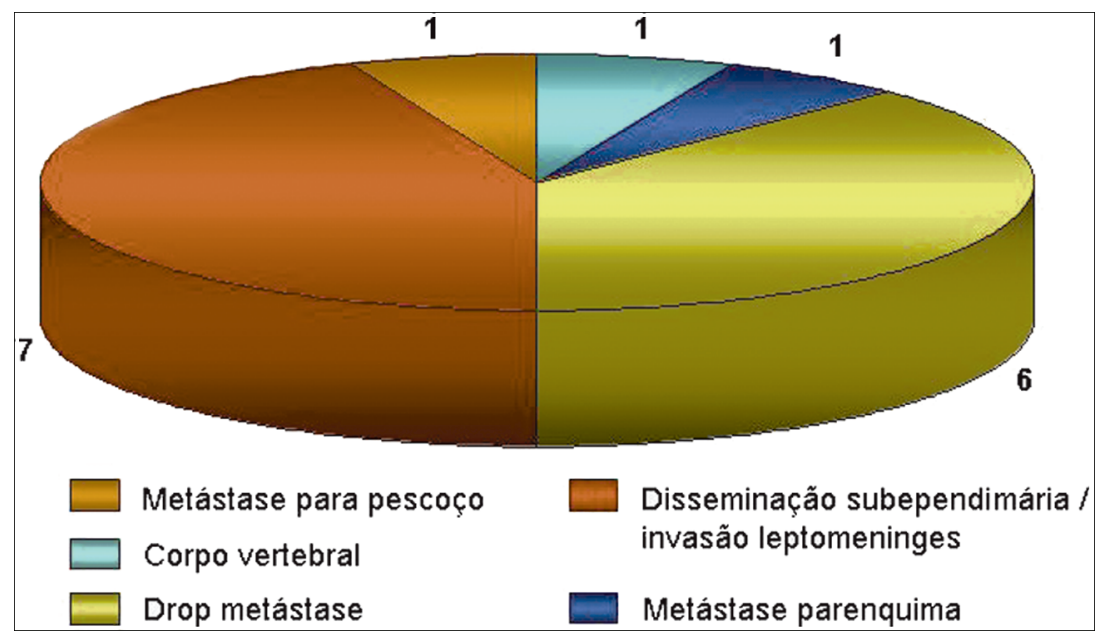

Gráfico 1.

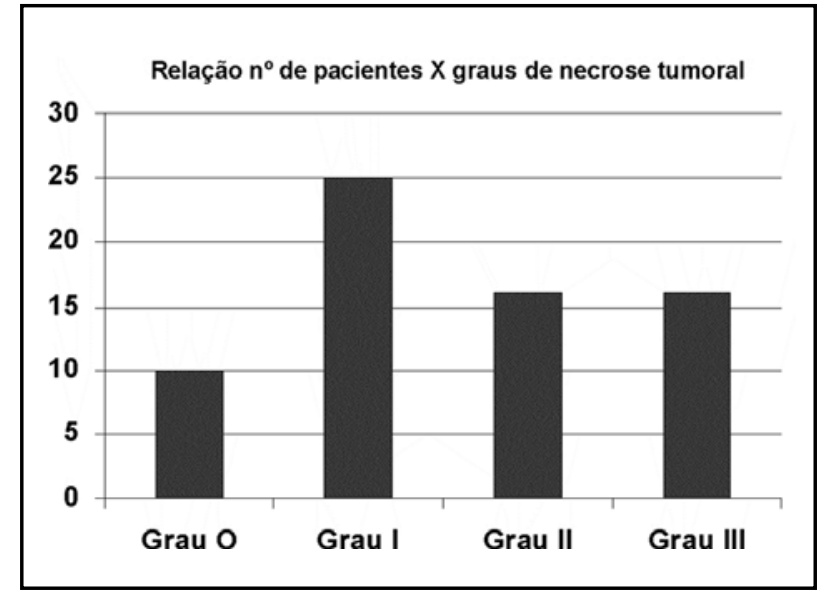

Gráfico 2. grau. Em $60 \%$ das áreas de edema freqüentemente observadas no GBM existem células tumorais ${ }^{1,2,4,5}$.

Microscopicamente apresentam uma variedade de padrões, tendo como mais comum células de tamanhos e formas variadas, células fibrilares, atividade mitótica intensa, proliferação vascular endotelial e necrose com áreas de hemorragia ${ }^{2,7,8}$.

Metástases extraneurais de GBM são raras, ape. sar destes serem altamente malignos ${ }^{3,7,8}$. Um tipo de metástase descrita neste tumor é a "drop" metástase, onde ocorre disseminação para o compartimento intra-raquiano por via subaracnóide/ leptomeningea ${ }^{5,6}$.

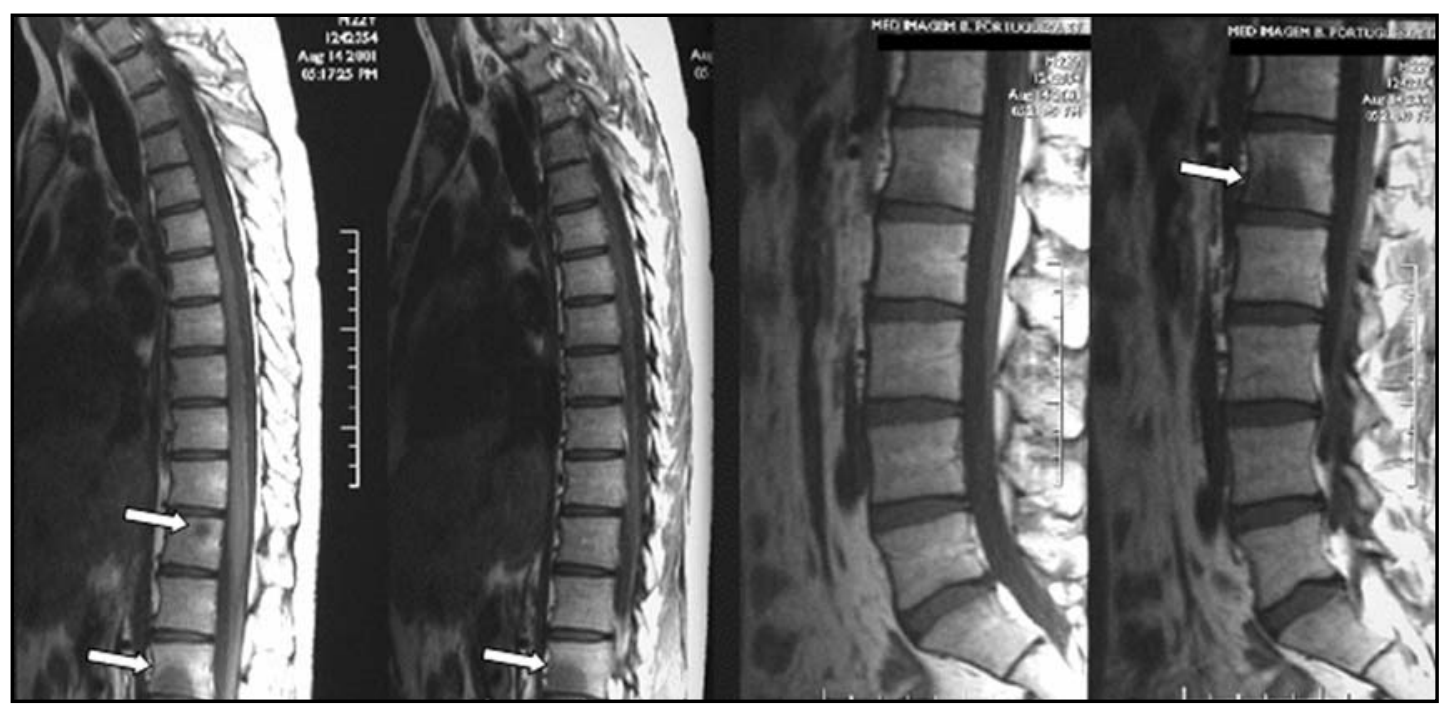

Fig 8. Sagital T1 das colunas dorsal e lombar, mostrando áreas hipointensas compatíveis com metástases nos corpos vertebrais de $D 9$, D11 e $L 1$. 
Os locais mais freqüentes de metástases extraneurais relatadas em ordem decrescente de incidência são: pulmão e pleura, linfonodos, osso e fígado $^{9,10}$.

A disseminação para pescoço também pode ser evidenciada em exames de seguimento da lesão

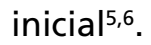

Em nossa pesquisa obtivemos dados concordantes com a literatura atual em relação à incidência por idade e localização no SNC. Embora a literatura destaque como raro o aparecimento de metástases, evidenciamos que $22 \%$ de nossos casos tiveram esta manifestação. Outro ponto discordante em relação à literatura foi a não detecção de implantes viscerais, pois encontramos apenas implante em linfonodo e osso.

O que pode explicar as discordâncias é o fato deste estudo ser mais atual que a literatura pesquisada e que nos dias atuais ocorreu avanço no tratamento radioterápico e quimioterápico, que em alguns casos aumenta a sobrevida destes pacientes e conseqüentemente os torna susceptíveis ao aparecimento de metástases e outras condições inerentes ao tratamento.

A literatura descreve que as metástases ocorrem mais comumente em pacientes que sofreram algum procedimento invasivo e que podem dar chance ao desprendimento de células tumorais ${ }^{3}$.

O grau de necrose tumoral é também um dado importante, apontado na literatura como sinal de mau prognóstico, juntamente com hemorragia e proliferação vascular ${ }^{1,2,4,5}$.
Em 67 casos estudados verificamos que 32 casos tinham graus de necrose II e III (48\%), e no restante dos casos os graus de necrose foram 0 e I ( $52 \%)$. Destes 32 casos com graus de necrose avançados $31,25 \%$ (10 casos) tiveram aparecimento de metástases enquanto que nos 35 casos com graus de necrose 0 ou I somente notou-se este fato em $7 \%$ ( 5 casos).

Em conclusão, a incidência de metástases nos nossos pacientes foi de $22 \%$, fato este que a literatura descreve como raro. Também o aparecimento de metástases foi maior na população de pacientes com mais altos graus de necrose ( II e III) do que quando comparamos aos pacientes que tinham graus de necrose inferiores ( 0 e l).

\section{REFERÊNCIAS}

1. Atlas SW, Magnetic resonance imaging of the brain and spine, 3rd ed. Philadelphia: Williams and Wilkins, 2002.

2. Berger PC, Scheithauer BW. Tumors of neuroglia and choroid plexus epithelium In Tumors of the central nervous system. AFIP Atlas of tumor pathology, 1993:25-161.

3. Gamis AS, Egelhoff J, Roloson G, et al. Diffuse bony metastases at presentation in a child with glioblastoma multiforme. Cancer 1990;66:180-184.

4. Lacroix M, Abi-Said D, Fourney DR, et al. A multivariate analysis of 416 patients with glioblastoma multiforme: prognosis, extent of resection and survival. J Neurosurg 2001;95:190-198.

5. Osborn A G. Diagnostic neuroradiology. St. Louis: Mosby, 1994.

6. Kuroiwa T, Numaguchi Y, Rothman MI, et al. Posterior fossa glioblastoma multiforme: MR findings. AJNR 1995; 16: 583-589.

8. Dolman CL. Lymph node metastasis as first manifestation of glioblastoma. J Neurosurg 1974;41:607-609.

9. El-Gindi S, Salama M, El-Henawy M, Farag S. Metastases of glioblastoma multifome to cervical lymph nodes. J Neurosurg 1973;38:631-634.

10. Myers T, Egelhoff J, Myers M. Glioblastoma multiforme presenting as osteoblastic metastatic disease. AJNR 1990;11:802-803.

11. Wallace CJ, Forsyth PA, Edwards DR. Lymph node metastases from glioblastoma multiforme. AJNR 1996;17:1929-1931. 